\title{
Propriospinal myoclonus in tetraplegic patients: clinical, electrophysiological and therapeutic aspects
}

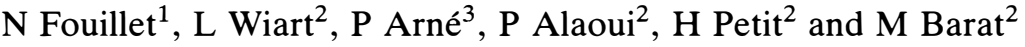 \\ ${ }^{1}$ Service de Neurologie, Centre Hospitalier de la Côte Basque, 64109 Bayonne Cedex; ${ }^{2}$ Service de Rééducation \\ Neurologique, Centre Hospitalier Pellegrin, 33076 Bordeaux Cedex; ${ }^{3}$ Service d'Exploration Fonctionnelle \\ Neurologique, Centre Hospitalier Pellegrin, 33076 Bordeaux Cedex, France
}

Propriospinal myoclonus is a rare and relatively little studied complication of spinal cord injury. We report two patients with an extension-producing myoclonus presenting with tetraplegia caused by cervical trauma. Rhythmic extension jerks of the trunk and lower limbs appeared several weeks after their injury in a context of severe spasticity. The characteristics of these jerks were determined by polymyography of 12 muscles. They lasted between 306 and $1127 \mathrm{~ms}$ with a frequency of 0.3 to $0.5 \mathrm{~Hz}$. By comparing latencies their origin was found to be in the lumbar cord from which there was a slow $\left(2 \mathrm{~m} \mathrm{~s}^{-1}\right)$ upward and downward spread. Oral treatment with baclofen and sodium valproate was partially successful in one patient, but ineffective in the other. Intrathecal 75 or $100 \mu \mathrm{g}$ baclofen produced a striking, complete disappearance of myoclonus prompting the implantation and successful use of a baclofen pump in one patient. These two new cases suggest the existence of a lumbar generator in which myoclonic extension jerks originate, and demonstrate a new therapeutic alternative in intrathecal baclofen for patients resistant to oral medication.

Keywords: myoclonus; spasticity; tetraplegia; baclofen

\section{Introduction}

Spinal myoclonus, initially described by Lhermitte, ${ }^{1}$ is a rare and still poorly understood complication of spinal cord lesions. ${ }^{2-4}$ Among these slow rhythmic abnormal movements, Brown ${ }^{3,4}$ distinguishes segmental myoclonus affecting a limited number of metameres, from propriospinal myoclonus involving a more extensive spinal region.

We present two patients with propriospinal myoclonus eliciting extension, a very rarely described form, to which electrophysiological studies and intrathecal baclofen trials were applied.

\section{Case 1}

A 27-year-old man, whose prior medical history was unremarkable, sustained a fracture-dislocation from a fall, of the 5th and 6th cervical vertebrae with immediate partial C5, complete C6 tetraplegia in December 1991. Emergency reduction and osteosynthesis were performed by an anterior approach. During the postoperative period, the patient developed cardiopulmonary failure and severe pneumonia necessitating 4 weeks in intensive care. Neurological status remained as a complete $\mathrm{C} 6$ tetraplegia.

Five weeks after the accident, slight predominately leftsided, bilateral slow rhythmic $(0.3 \mathrm{~Hz})$ jerks appeared, affecting the extensor muscles caudal to the injury: erector spinae, gluteals, quadriceps femoris, adductors, and gastro- cnemius. These jerks were constant, and were enhanced by voluntary spreading of the arms and by stimulation of the skin and viscera caudal to the lesion. They were inhibited by flexion and lateral rotation of the legs (lotus position), and by sleep. The patient had substantial spasticity and rigidity with flexion or extension spasms induced by cutaneous stimulation which would temporarily mask the jerks.

Over the course of the next 12 months, the sensory and motor deficits remained stable, but the myoclonus increased, becoming painful and functionally disabling. The jerks spread to segments adjacent to those of the lesion, less intensely affecting the pectorals, triceps and finger extensors. Radiographs and MRI studies showed neither compression nor the development of additional cord lesions.

The following electrophysiological parameters were investigated.

1 Back average EEG revealed no simultaneous bursts of cortical activity during myoclonic jerks.

2 Computer-assisted polymyography of 12 muscles recorded during $2700 \mathrm{~ms}$ (Table 1, Figures 1 and 2) showed a spontaneous rhythmic muscular activity of 0.3 to $0.5 \mathrm{~Hz}$ consisting of trains ranging in duration from 306 to $1014 \mathrm{~ms}$ as well as a chronological sequence of muscular activation beginning in the upper lumbar area, then spreading simultaneously to the lower lumbar and sacral segments in $165 \mathrm{~ms}$ and to the thoracic and cervical segments in $162 \mathrm{~ms}$.

3 Motor evoked potentials recorded at level L4 after magnetic stimulation at level C7 were normal, indicating good medullary conduction between levels C7 and L4. 
Table 1 Polymyography: duration and recruitment order

\begin{tabular}{|c|c|c|c|c|}
\hline \multirow[t]{2}{*}{ Muscles examined } & \multicolumn{2}{|c|}{ Case 1 (C6 level) } & \multicolumn{2}{|c|}{ Case 2 (C8 level) } \\
\hline & $M D A P(m s)$ & $M D L(m s)$ & $M D A P(m s)$ & $M D L(m s)$ \\
\hline Left pectoralis major $(\mathrm{C} 5-\mathrm{T} 1)$ & 555 & 162 & & \\
\hline Left extensor digitorum $\mathrm{V}$ proprius $(\mathrm{C} 7-8)$ & 306 & 158 & & \\
\hline Right extensor digitorum $\mathrm{V}$ proprius $(\mathrm{C} 7-8)$ & 497 & 136 & & \\
\hline Left thoracic paraspinals (T6-8) & 737 & 128 & 1100 & 63 \\
\hline Right thoracic paraspinals (T6-8) & 791 & 120 & 1169 & 41 \\
\hline Right pectoralis major (C5-T1) & 869 & 93 & & \\
\hline Right lumbar paraspinals (L1-5) & 1014 & 0 & 1099 & 0 \\
\hline Left lumbar paraspinals (L1-5) & 737 & 32 & 1067 & 37 \\
\hline Right adductor (L1-2) & 883 & 95 & 1127 & 42 \\
\hline Right gluteus maximus (L5-S2) & 801 & 106 & 992 & 183 \\
\hline Left adductor (L2-4) & 712 & 124 & 1130 & 83 \\
\hline Right vastus medialis (L2-4) & & & 989 & 98 \\
\hline Left vastus medialis (L2-4) & & & 902 & 129 \\
\hline Left gluteus maximus (L5-S2) & 641 & 165 & 969 & 196 \\
\hline Left soleus (L5-S2) & & & 916 & 202 \\
\hline Right soleus (L5-S2) & & & 812 & 232 \\
\hline
\end{tabular}

MDAP: mean duration of the action potentials; MDL: mean difference in latency of myoclonus

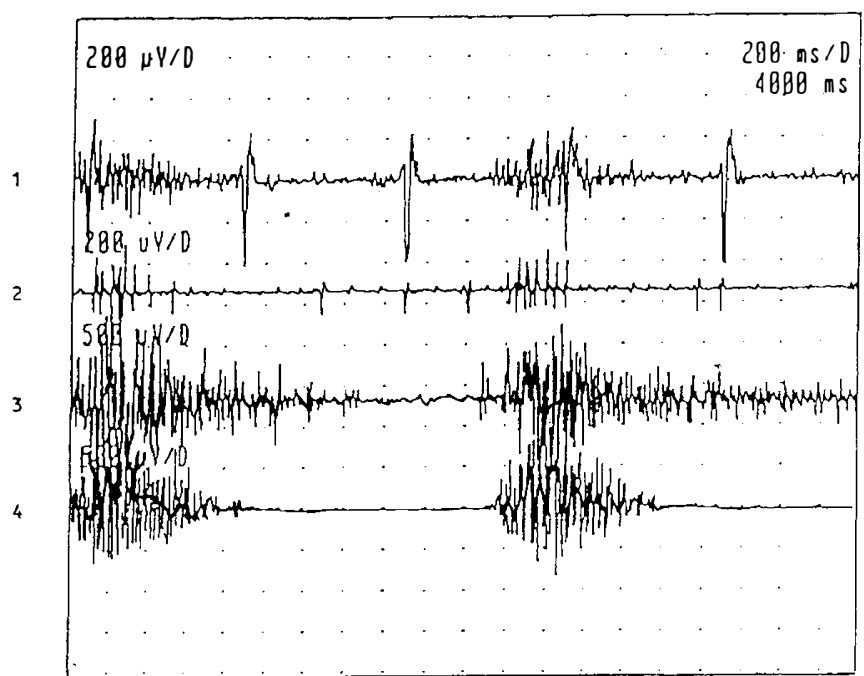

Figure 1 Polygraphy (case 1). Example of easily distinguishable rhythmic activity. Muscles: (1) pectoralis major; (2) extensor digitorum V proprius; (3) thoracic paraspinals; (4) adductor

4 Electromyography at the level of the spinal injury showed non-specific denervation.

Intrathecal administration of baclofen by lumbar puncture was then tested. With $50 \mu \mathrm{g}$, the patient's rigidity disappeared as did the tendon reflexes in the lower, but not in the upper limbs. Spontaneous myoclonus was also absent, but external stimulation produced weak rhythmic bursts progressively disappearing over $20 \mathrm{~s}$. These effects lasted approximately $10 \mathrm{~h}$. With $100 \mu \mathrm{g}$, all of the symptoms abated for $24 \mathrm{~h}$.

Whilst waiting for a baclofen pump implantation, the patient was treated with $90 \mathrm{mgday}^{-1}$ baclofen and 1,500 $\mathrm{mgday}^{-1}$ sodium valproate orally, resulting in a partial attenuation of the symptoms, satisfying the patient. One

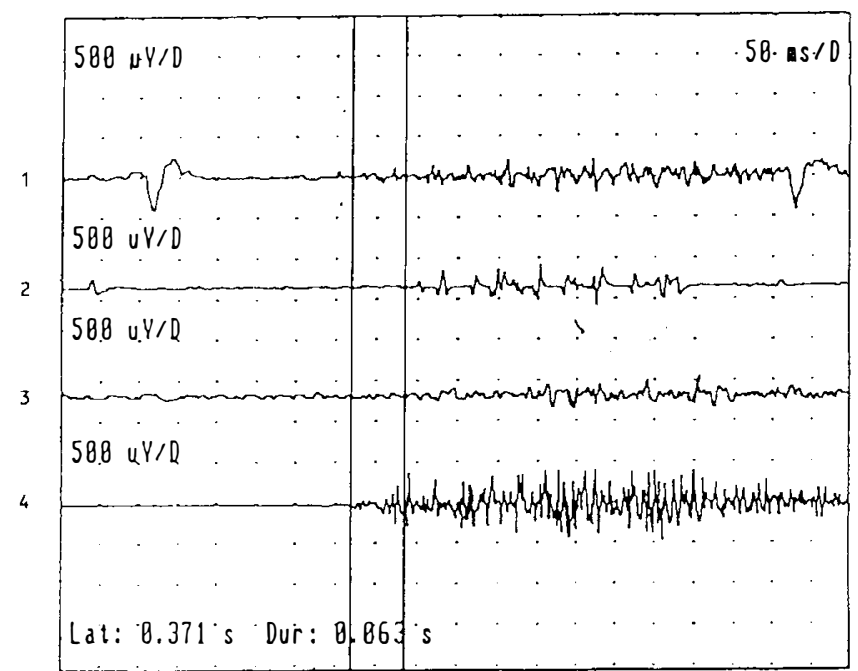

Figure 2 Polygraphy (case 1). Example of measurement of difference in latencies. Muscles: (1) pectoralis major; (2) extensor digitorum $\mathrm{V}$ proprius; (3) thoracic paraspinals; (4) adductor

year later (February 1994) the situation remained stable and the oral treatment was maintained.

\section{Case 2}

During a motorcycle accident in February 1992, this 31-year-old man, with no notable antecedent medical history, sustained a slight head injury (brief loss of consciousness), multiple fractures of the upper and lower limbs, and a fracture-dislocation of the 7th and 8th cervical vertebrae producing an immediate C7 tetraplegia. After emergency reduction and osteosynthesis, the postoperative period was complicated by cardiopulmonary failure necessitating mechanical ventilatory support in the intensive care unit for 8 
680

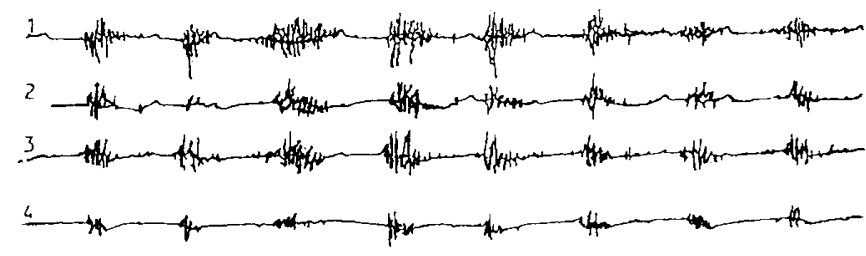

5
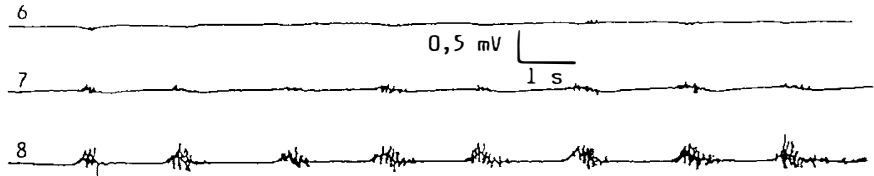

9

10

Figure 3 Polygraphy (case 2). Example of spontaneous rhythmic activity. Muscles: (1) right thoracic paraspinals; (2) left thoracic paraspinals; (3) right lumbar paraspinals; (4) left lumbar; (5) right glutens maximus; (6) left gluteus maximus; (7) right adductor; (8) left adductor; (9) right soleus; (10) left soleus
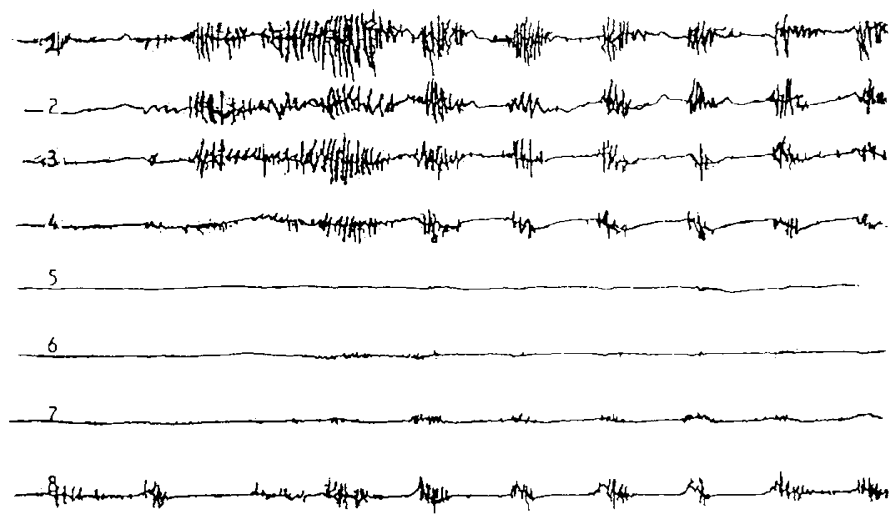

$-9$

$-10$

Figure 4 Enhancement of rhythmic activity and appearance of tonic activity between bursts observed during painful stimulation (foot pitching). Muscles: (1) right thoracic paraspinals; (2) left thoracic paraspinals; (3) right lumbar paraspinals; (4) left lumbar; (5) right gluteus maximus; (6) left gluteus maximus; (7) right adductor; (8) left adductor; (9) right soleux; (10) left soleus

days. During the following 8 months the patient developed severe flexion and extension spasms of the trunk and lower limbs which resisted orally administered baclofen $\left(90 \mathrm{mg} \mathrm{day}^{-1}\right)$ and diazepam.

After a satisfactory trial of intrathecal baclofen $(50 \mu \mathrm{g})$ in November 1992, a pump implantation was programmed for the following spring. In May 1993, on an unchanged background of spasticity, he developed rhythmic, 0.2 to $0.5 \mathrm{~Hz}$ jerks of the extensor muscles of the trunk and lower limbs, but sparing the upper limbs. These episodes of myoclonus were sporadic, lasting from a few minutes to several hours, of varying intensity, and occurred predomi- nately in the morning and late afternoon. They were induced by urinary-tract infections, turning of the patient, transfer manoeuvres, and by skin stimulation, below the lesion.

A radiological and $\mathrm{MR}$ assessment revealed no new abnormalities in the region of the spinal injury.

The following electrophysiological examinations were performed at that time.

1 Back average EEG, measuring frontal activity during EMG, showed no activity during myoclonus thereby eliminating any possible cortical origin.

2 Computer-assisted polymyography of 13 muscles recorded for periods of $2700 \mathrm{~ms}$ demonstrated a slow rhythmic activity $(0.3$ to $0.5 \mathrm{~Hz})$ of all the muscles examined, sometimes tapering off spontaneously, and enhanced by cutaneous stimulation. Activation lasted from $812 \mathrm{~ms}$ (soleus) to $1130 \mathrm{~ms}$ (adductors) (see Table 1, Figures 3 and 4), and appeared to have the same rhythms with slightly different delays. This muscular activation began in the lumbar region, then simultaneously spread toward the thoracic and sacral segments in $63-232 \mathrm{~ms}$.

3 Somatosensory evoked potentials after stimulation of tibial nerves showed a diminished and delayed response at $60 \mathrm{~ms}$ on the left and no response on the right.

Intrathecal administration of $75 \mu \mathrm{g}$ of baclofen completely abolished myoclonus, spasticity, and tendon reflexes for over $12 \mathrm{~h}$. The intrathecal infusion of $100 \mu \mathrm{g}$ day ${ }^{-1}$ from an implanted pump (a $30 \mu \mathrm{g}$ bolus at $6 \mathrm{am}$ and $5 \mathrm{pm}$ with a constant interbolus rate of $40 \mu \mathrm{g} 24 \mathrm{~h}^{-1}$ ) maintained these effects. Currently, after $1 \frac{1}{2}$ years follow-up, it has not been necessary to increase this dosage.

\section{Discussion}

There has been a renewal of interest in propriospinal myoclonus following recent reports. ${ }^{2,3,5}$ This syndrome develops after lesions of the cord usually involving the cervical portion ${ }^{3}$ as in our two patients. Rarely, the initial lesion is to the thoracic or lumbar cord. ${ }^{2}$ Initial cord damage can be traumatic, ${ }^{2-4}$ ischaemic, ${ }^{6}$ inflammatory ${ }^{7}$ or infectious. ${ }^{8}$ Several examples of undetermined aetiology ${ }^{3}$ have however been reported.

A typical clinical picture is one of slow rhythmic $(0.3$ to $0.7 \mathrm{~Hz})$ bilateral, synchronous jerks of the muscles of flexion in the trunk and lower limbs.

Brown $^{4}$ recently described a clinical picture of alternating jerks of the axial flexors and extensors, resulting in a veritable dance of the trunk. Cases predominately involving rhythmic jerks of the extensors alone as was seen in our patients are much rarer. ${ }^{2,6}$

A better comprehension of the pathophysiology of this syndrome has resulted from recent electrophysiological analyses employing computer-assisted polymyography and determination of latencies. ${ }^{2-5}$ Cortical participation in this type of myoclonus can be eliminated using back average EEG. ${ }^{3-5}$ This was performed in our patients in spite of the complete nature of their cord lesions in order to affirm, without any doubt, the spinal origin of these jerks. Computer-assisted polymyography is the ideal means of investigating this syndrome, permitting a simultaneous recording of more than a dozen muscles and a comparison of their activation latencies. These bursts of activation last from 
40 to $1500 \mathrm{~ms}$ according to the number of fibres activated. Their frequency ranges from 0.3 to $0.7 \mathrm{~Hz}$. This type of examination can establish the chronological order of muscle activation, which is impossible to determine clinically. Using this technique, Bussel, ${ }^{2}$ then Brown ${ }^{3,4}$ determined the presence of a focus of activation in the thoracic cord. The spread from this spinal generator to the upper- and lower-lying metameres is associated with flexion myoclonus. The very slow transmission (from 2 to $10 \mathrm{~m} \mathrm{~s}^{-1}$ ) suggests a polysynaptic transmission by propriospinal pathways. ${ }^{5}$ According to the same authors, ${ }^{2,3}$ such foci of activation are more likely to represent a physiological generator released by a more rostrally lying spinal lesion than to a zone of neuronal damage.

In our two patients, we were able to demonstrate, for the first time, the existence of this type of slow symmetric up- and downward spread of muscular activation originating in a lumbar focus.

Bussel $^{2}$ suggested that such a focus might participate in the generation of complex spinal patterns of locomotion analagous to those identified in animals. ${ }^{8}$

The electrophysiological characteristics of the myoclonus presented by our patients resemble those reported in the literature with an activation duration ranging from 306 to $1127 \mathrm{~ms}$, a frequency between 0.3 and $0.5 \mathrm{~Hz}$, and a diffusion speed estimated to be $2 \mathrm{~m} \mathrm{~s}^{-1}$.

Aside from aetiological treatment, the management of this syndrome includes the oral administration of baclofen or of anticonvulsant drugs (carbamazepine, clonazepam, sodium valproate), with variable results. $^{2-5,9}$ Our two subjects illustrate this well. Case 2 failed to respond to baclofen, clonazepam, and carbamazepine. In case 1 , baclofen associated with sodium valproate was only partially effective.

Jeon ${ }^{10}$ reported good results after continuous intrathecal infusion of morphine. The use of intrathecal baclofen is reported for the first time in this condition.
The remarkable effectiveness of baclofen in moderate doses $(75-100 \mu \mathrm{g})$, and the persistence of this action over time (follow-up of $1 \frac{1}{2}$ years in case 2 ) are noteworthy, and emphasise the importance of interneurons in the transmission of the trains of activation.

In case 1 , the persistence of monosynaptic reflexes in the upper limbs after the disappearance of myoclonus and reflexes in the legs is a supplementary argument in favour of a caudal focus of activation.

In conclusion, this work supports the hypothesis of a lumbar focus which produces extending propriospinal myoclonus, as opposed to flexion myoclonus which is considered to have a thoracic origin. Intrathecal baclofen offers a new therapeutic possibility when oral treatment fails.

\section{References}

1 Lhermitte J. La Section Totale de la Moelle Dorsale. Tardy Pigelet: Bourges, 1919, pp 30 and 110.

2 Bussel B et al. Myoclonus in a patient with spinal cord transection. Brain 1988; 111: 1235-1245.

3 Brown P, Rothwell JC, Thompson PD, Marsden CD. Propriospinal myoclonus. Evidence for spinal pattern generators in humans. Movement Disord 1994; 9: 571-576.

4 Brown $\mathrm{P}$ et al. Axial myoclonus of propriospinal origin. Brain 1991; 114: 119-214.

5 Chokzoverty S, Walter A, Zimmerman T, Picone M. Propriospinal myoclonus: a neurophysiologic analysis. Neurology 1992; 42: 1591-1595.

6 Djindjian R, Mamo H, Saimot G, Houdart R. Contractures tétaniformes paroxystiques au cours de l'artériographie sélective de l'artère d'Adamkiewicz. Presse Médicale 77: 1311-1314.

7 Kapoor R, Brown P, Thompson PD, Miller DH. Propriospinal myoclonus in multiple sclerosis. J Neurol Neurosurg Psychiatry 1992; 55: 1086-1088.

8 Grillner S. Locomotion in vertebrates: central mechanisms and reflex interaction. Physiol Rev 1975 55: 247-304.

9 Jankovik J, Pardo R. Segmental myoclonus clinical and pharmacologic study. Arch Neurol 1986; 43: 1025-1031.

10 Jeon B, Lee M, Erickson D. Successful treatment of medically unresponsive spinal myoclonus with continuous intrathecal morphine administration (abstract). Neurology 1991; 41: 416. 